\title{
Electrical impedance myography as a biomarker to assess ALS progression
}

\section{Citation}

Rutkove, Seward B., James B. Caress, Michael S. Cartwright, Ted M. Burns, Judy Warder, William S. David, Namita Goyal, et al. 2012. "Electrical Impedance Myography as a Biomarker to Assess ALS Progression." Amyotrophic Lateral Sclerosis 13 (5) (June 7): 439-445. doi:10.3109/17482968.2012.688837.

\section{Published Version}

10.3109/17482968.2012.688837

\section{Permanent link}

http://nrs.harvard.edu/urn-3:HUL.InstRepos:33973667

\section{Terms of Use}

This article was downloaded from Harvard University's DASH repository, and is made available under the terms and conditions applicable to Other Posted Material, as set forth at http:// nrs.harvard.edu/urn-3:HUL.InstRepos:dash.current.terms-of-use\#LAA

\section{Share Your Story}

The Harvard community has made this article openly available.

Please share how this access benefits you. Submit a story.

\section{Accessibility}




\title{
Electrical impedance myography as a biomarker to assess ALS progression
}

\author{
Seward B. Rutkove ${ }^{1}$, James B. Caress ${ }^{2}$, Michael S. Cartwright ${ }^{2}$, Ted M. Burns ${ }^{3}$, Judy \\ Warder $^{3}$, William S. David ${ }^{4}$, Namita Goyal ${ }^{4}$, Nicholas J. Maragakis ${ }^{5}$, Lora Clawson ${ }^{5}$, \\ Michael Benatar ${ }^{6}$, Sharon Usher ${ }^{7}$, Khema R. Sharma ${ }^{6}$, Shiva Gautam ${ }^{8}$, Pushpa \\ Narayanaswami ${ }^{1}$, Elizabeth M. Raynor ${ }^{1}$, Mary Lou Watson ${ }^{9}$, and Jeremy M. Shefner ${ }^{9}$
}

${ }^{1}$ Department of Neurology, Beth Israel Deaconess Medical Center, Boston, Massachusetts 2Department of Neurology, Wake Forest School of Medicine, Winston-Salem, North Carolina ${ }^{3}$ Department of Neurology, University of Virginia, Charlottesville, Virginia ${ }^{4}$ Department of Neurology, Massachusetts General Hospital, Massachusetts ${ }^{5}$ Department of Neurology, Johns Hopkins Medical Center, Baltimore, Maryland ${ }^{6}$ Department of Neurology, University of Miami, Miami, Florida ${ }^{7}$ University of South Florida, Tampa, Florida ${ }^{8}$ General Clinical Research Center, Beth Israel Deaconess Medical Center, Boston, Massachusetts ${ }^{9}$ Department of Neurology, Upstate Medical Center, Syracuse NY, New York, USA

\section{Abstract}

Electrical impedance myography (EIM), a non-invasive, electrophysiological technique, has preliminarily shown value as an ALS biomarker. Here we perform a multicenter study to further assess EIM's potential for tracking ALS. ALS patients were enrolled across eight sites. Each subject underwent EIM, handheld dynamometry (HHD), and the ALS Functional Rating Scalerevised (ALSFRS-R) regularly. Techniques were compared by assessing the coefficient of variation $(\mathrm{CoV})$ in the rate of decline and each technique's correlation to survival. Results showed that in the 60 patients followed for one year, EIM phase measured from the most rapidly progressing muscle in each patient had a $\mathrm{CoV}$ in the rate of decline of 0.62 , compared to HHD (0.82) and the ALSFRS-R (0.74). Restricting the measurements to the first six months gave a CoV of 0.55 for EIM, 0.93 for HHD, and 0.84 for ALSFRS-R. For both time-periods, all three measures correlated with survival. Based on these data, a six-month clinical trial designed to detect a $20 \%$ treatment effect with $80 \%$ power using EIM would require only 95 patients/arm compared to the ALSFRS-R, which would require 220 subjects/arm. In conclusion, EIM can serve as a useful ALS biomarker that offers the prospect of greatly accelerating phase 2 clinical trials.

\section{Keywords}

Clinical trials; amyotrophic lateral sclerosis; electrical impedance myography

\section{Introduction}

Phase 2 trials in ALS are limited by the lack of a sensitive marker of disease progression (1). To date, the ALS Functional Rating Scale-revised (ALSFRS-R) (2), strength measurements via handheld dynamometry (HHD), functional vital capacity, and motor unit number

(C) 2012 Informa Healthcare

Correspondence: S. B. Rutkove, Division of Neuromuscular Diseases, Beth Israel Deaconess Medical Center, 330 Brookline Avenue, TCC-810, Boston, MA 02215, USA. Fax: 617-667-3175. srutkove@ bidmc.harvard.edu. 
estimate (MUNE) are being used, although the ALSFRS-R has generally become the preferred tool (3-5).

One newer approach that may be useful for following disease progression and potential response to treatment is the technique of electrical impedance myography (EIM) (6). In EIM, a high frequency, low-intensity electrical current is applied to a limb, and the resulting voltages are measured over a discrete region of muscle. The basic hypothesis is that changes in the structure and composition of the muscle with disease will be reflected in alterations in the localized impedance of the muscle. Unlike standard electrophysiologic techniques, EIM does not measure the inherent electrical properties of the muscle but rather the effect of the tissue on the applied current. In addition to showing abnormalities in a variety of other neuromuscular conditions including myositis (7), spinal muscular atrophy (8), and disuse atrophy (9), a single-center study found that EIM was a sensitive indicator of ALS progression (10); a subsequent study in ALS SOD1-G93A rats confirmed those findings (11). Advantages of EIM include that it is non-invasive, painless, rapid to apply, requires minimal training, is highly reproducible (12), and can be applied to virtually any superficial muscle.

In this study, we assessed EIM's potential value as a biomarker of disease progression by following a group of ALS patients over time and comparing EIM to HHD and ALSFRS-R.

\section{Material and methods}

\section{Standard protocol approvals, registrations, and patient consents}

The procedures followed were in accordance with the ethics standards of the responsible committee on human experimentation and with the Helsinki Declaration of 1975, as revised in 1983 and as overseen by the institutional review boards of all participating institutions; in addition, written informed consent was obtained from all subjects. The study was performed at eight sites (principal investigator initials in parentheses): Beth Israel Deaconesss Medical Center (SR), Massachusetts General Hospital (WD), Upstate Medical Center (JS), Johns Hopkins University (NM), University of Virginia (TB), Wake Forest University (JC, MC), Emory University (MB), and University of Miami (SK).

\section{Overall study design}

This investigation was designed as a natural history study of ALS using EIM, HHD and ALSFRS-R, with no planned interventions. Modified incremental MUNE was also included in the study protocol; the results of that analysis have been reported previously (13). The goal was to follow individuals for approximately one year with visits spaced at a maximum of approximately three months apart; however, more frequent visits were encouraged.

\section{Subjects}

Participants were recruited consecutively. Inclusion criteria included: 1) age 18-85 years at time of enrollment; 2) a history of probable, probable-laboratory supported (as defined by the presence of fibrillation potentials in clinically unaffected limbs), and definite ALS by the modified El Escorial criteria (14) or a positive gene test for a form of familial ALS; 3 ) disease duration for no longer than five years from diagnosis; 4) FVC > 60\%; and 5) average limb muscle strength of greater than 3.5 on a modified manual muscle testing scale.

Exclusion criteria included: 1) presence of superimposed neuromuscular disease; 2) presence of implanted pacemaker or other electronic device. 


\section{Training, validation, and quality assurance}

Prior to study initiation, all evaluators were taught the techniques of EIM and HHD at a single training session. All evaluators were required to demonstrate test-retest repeatability for EIM and HHD with less than 15\% variation for each individual muscle studied in four healthy individuals with repeated measurements being at least $3 \mathrm{~h}$ apart. Any new research team members were required to be trained in the techniques and demonstrate similar reproducibility. A single site visit was also made to all centers to help ensure accurate data collection.

\section{EIM}

There are a number of approaches for performing EIM, both from the choice of impedancemeasuring device to electrode positioning. Given the multicenter nature of the study and the need for a cost effective measure, rather than utilizing a multifrequency system as has been the case in more recent work $(8,11,15)$, we used the DF50 ${ }^{\circledR}$ (Impedimed, Inc, San Diego, CA). This instrument provides a numerical readout of the electrical impedance data (resistance, reactance, phase, and impedance) at a single frequency of $50 \mathrm{kHz}$. Based on the results of the earlier ALS study (10), we chose the $50-\mathrm{kHz}$ phase as the primary measure to follow.

Since ALS does not typically affect the whole body at once but rather spreads regionally (16), we studied several muscles in each patient with the hope of capturing a muscle that was most actively deteriorating during the study period. In order to assist with this, if a patient was minimally weak in one arm and markedly weak in the other, the minimally weak arm would be chosen with the expectation it would show greater decline during the study period. In addition, since we were performing EIM using a far-current electrode approach (17), limbs with joint or knee replacements were not studied. Thus, the following muscles were measured unilaterally: biceps, wrist extensors, abductor pollicis brevis or abductor digiti quinti (abductor pollicis brevis was chosen, unless a median neuropathy was identified, in which case abductor digiti quinti was chosen), quadriceps, and tibialis anterior. Figure 1 shows an example of the measurements being performed on the forearm extensor compartment.

In order to ensure accurate electrode placement from visit to visit, predetermined specific anatomic landmarks were used for electrode placement. The use of pinpoint tattoos was also encouraged, although not employed routinely at all centers.

Intra-session repeatability was assessed for biceps brachii by repeating an identical set of measurements at the completion of the entire EIM measurement session (i.e. biceps was studied at the beginning and then again at the end, with the other EIM measurements performed in between). Standard whole-body bioimpedance measurements were also made on both sides (18), since such measurements also alter to some extent with ALS progression (19).

\section{Handheld dynamometry}

Handheld dynamometry was performed with the MicroFet2 (Hoggan Industries, Logan, UT) across all sites, using standard measurement technique of multiple upper and lower extremity muscles. Mean values were calculated for each visit and used in the analyses.

\section{ALSFRS-R}

The ALSFRS-R was completed at the time of each visit with the assistance of the study coordinator or research assistant. 


\section{Data analysis}

The main goal of this study was to identify each technique's capability to assess decline in the group of individuals over time. One convenient measure of this is the coefficient of variation $(\mathrm{CoV})$ of the rate of decline across a group of individuals (the standard deviation in the rate of decline/mean rate of decline). Put simply, the lower the value of the CoV the more powerful the potential biomarker. Thus, for each patient with a minimum of three visits, random intercept and slope terms were specified within a linear mixed model set to obtain slopes of each measure. Variances of slopes were obtained by specifying an unstructured covariance configuration. In the few situations where the model did not converge, the intercept was dropped from the model and therefore not estimated. From these estimates, coefficients of variation of the slopes were estimated for EIM, HHD, and ALSFRS-R. No data points were imputed.

Two EIM values were assessed: the mean EIM 50-kHz phase slope from the five muscles and the steepest single-muscle slope from each patient. Mean EIM phase slope was computed by averaging the individual slopes of five muscles. Variance of the mean slope was computed assuming the independence of the single muscle EIM phase values so that if $V_{i}$ is the variance of the EIM phase slope used in calculation of the mean slope then standard deviation of the mean slope $=\frac{1}{5} \sqrt{\Sigma V_{i}}$. The steepest slope of decline in phase was also computed. For this measure, linear regression was used to obtain the EIM phase slope over time for each of the five muscles. Subsequently, for each patient, the steepest (most negative) slope was identified from each of the five individual muscle slopes. For ALSFRS$\mathrm{R}$, the simple summed score was used to calculate the slope of decline. For HHD, the average value of all measured muscles was used, although a 'steepest slope' approach, analogous to that used for EIM, was also evaluated. Cox proportional hazards analysis was used to assess the relationship between rate of decline and survival across patients. Finally, in order to determine how well each individual measure would perform in a hypothetical clinical trial, a power calculation was performed as previously described using a random effects model (10). Unless otherwise specified, results are given as mean $+/-$ standard deviation.

\section{Results}

\section{Enrollment and demographics}

From a total of 89 subjects, 60 had three or more visits with complete or near-complete data sets. The majority of subjects who had less than three visits failed the screening visit or did not return for testing after the first measurement session; a smaller proportion had only two full visits. The proportion of males to females was unexpectedly elevated at 3:1 with a mean age of 56.1 +/- 12 years. The mean number of visits was 5.1 (range 3-11) and the mean length of follow-up was 304 days (range 94-501). Mean disease duration at the time of enrollment from the onset of symptoms was $2.5+/-1.8$ years. Nine of the 60 subjects (15\%) had bulbar-onset disease, a proportion just slightly lower than that reported in other recent trials $(3,20,21)$.

\section{EIM repeatability}

The mean intra-session between-measurement variation in biceps phase was $11.9 \%$ with an intraclass correlation coefficient of 0.90 .

\section{EIM alterations over time compared to HHD, and ALSFRS-R}

Table I provides the CoVs and their 95\% confidence limits (22) in the rate of decline both after one year and after six months. At one year, EIM (both mean and steepest), HHD, and 
ALSFRS-R all had CoVs well below 1.0, although EIM steepest slope fared best, with a $\mathrm{CoV}$ of 0.62 ; this result also compared favorably to that identified for MUNE of 0.72 as previously reported (13). At six months, the steepest slope EIM values had an even lower $\mathrm{CoV}$ of just 0.55 ; the three other parameters, while still less than 1 , were considerably higher. Whole-body bioimpedance analysis data from each side showed very high coefficients of variation in the rate of decline for phase (over 4.0) and was not evaluated further. Figure 2 shows the six-month trajectories of the EIM data (both the steepest slope/ patient and the mean value/patient), ALSFRS-R and HHD for each for each the 50 patients who had three or more visits within the first six months of study are in gray; the thick black line in each plot represents the group mean values. The plots are normalized to each patient's baseline visit for ease of comparison.

These values are translated into patient numbers in Table II, which shows the number of subjects needed, based on the six-month data, for a hypothetical clinical trial as described previously (10), assuming an $80 \%$ power to detect a treatment effect of $20 \%$, at alpha $=$ 0.05 , one-tailed.

\section{Correlation with survival}

Twenty-four of the 60 subjects died during follow-up, 21 due to respiratory insufficiency secondary to ALS, three due to other causes (two myocardial infarctions and one head injury); these latter subjects' data were not considered ALS deaths and censored accordingly. Table III shows the Cox proportional hazards regression correlating the four parameters both at one year and at six months. At one year and at six months, steepest EIM phase, ALSFRS-R, and HHD all showed a significant correlation to the likelihood of death from respiratory insufficiency, although mean EIM phase did not.

\section{Discussion}

This study demonstrates the potential power of EIM to assess ALS progression in just six months, confirming and extending the original 2007 observation (10). Moreover, it demonstrates that the technique itself is clinically meaningful in that the rate of decline in steepest EIM phase, like ASLFRS-R and HHD, correlates to survival. The data also suggest that EIM may outperform the modified incremental MUNE method for tracking ALS progression (13), although MUNE remains a more direct measure of motor neuron loss.

One unusual feature of the analysis is our use of a single muscle to represent the overall rate of progression in each patient. However, using a single muscle to indicate the status of the entire individual is not novel as it is the underlying assumption of MUNE. By assessing the most rapidly progressing muscle (i.e. the one with the steepest slope) from each patient, we are able to maximize the rate of progression for the analysis and thus further improve the $\mathrm{CoV}$. The reason we believe this approach works is that ALS does not typically affect the entire body simultaneously, but rather spreads regionally (16). Most measures, such as the ALSFRS-R and HHD, rely on data from many body regions to reduce random noise, providing a rate of change for the patient as a whole. However, this has the untoward effect of diluting out the detection of disease progression since relatively unchanging regions both those that are end-stage and those that are only early in the disease course - will reduce the mean rate of decline and hence increase the CoV. With EIM it is possible to obtain highly repeatable data from one muscle (12), and thus safely employ single muscle data in the analysis. In contrast, for example, utilizing a single-muscle 'steepest slope' approach for HHD does not work because the technique is far less reliable; an identical analysis using single muscle HHD data gave high CoVs in the rate of decline of 2.0 at year and 1.5 at six months. With MUNE, one is restricted to measuring the same one or two muscles in each 
patient, and therefore no attempt can be made to identify the region that is most rapidly deteriorating during the study period.

This single muscle approach has to be meaningful, however, and that the rate of progression from this muscle correlates with survival in this group of individuals supports its validity as a potential biomarker. Whereas both HHD and ALSFRS-R had stronger correlations to survival both at six months and at one year than EIM, given that this biomarker would likely be used in phase 2 clinical trials where initial evidence of potential efficacy is sought, the trade-off for higher sensitivity to decline versus a correlation to survival is likely acceptable. Why the mean EIM rate of decline showed no relationship with survival is not clear and requires further study, although limitations with EIM measurement technique (discussed below) could have contributed. One limitation of the single muscle analysis approach is that coexistent compression neuropathies or radiculopathy could confound the data. While the former were excluded for ulnar- and median-innervated muscles, concomitant radiculopathy was not.

We found the mean intra-session variation in EIM phase measuring biceps was $11.9 \%$, which was considerably higher than the value of $4.71 \%$ we previously reported in normal subjects over more extended periods of time (12). This worse value likely reflects several different factors, including lack of regular practice in performing the technique given the relatively few patients enrolled at each site and limitations of the device utilized, compared to the system utilized in that previous study. The device used provided only a numerical read-out, and the accuracy of the data could not be verified except by repeating the measurement several times to confirm its stability. Moreover, the device is not calibrated to the lower impedance values encountered in such localized measurements, being designed for whole-body bioimpedance measurements. Assessing the two separate components of the phase, the reactance and resistance, helps highlight the device/measurement limitation. The reactance is about 1/10th the magnitude of the resistance and is thus more sensitive to noise. Consequently, the reactance values varied by $12 \%$ on average; in contrast, the more easily obtained resistance varied by only $3 \%$. Accordingly, improved instrumentation would likely further enhance the reproducibility of the technique and possibly its sensitivity to ALS progression.

One unexpected finding of this study was that the CoV for ALSFRS-R was unusually low (0.74 at one year, and 0.84 at six months). Another, smaller natural history study also evaluated change in the ALSFRS-R over time and showed a similarly low CoV of 0.76 (23). However, placebo groups from past clinical trials have shown consistently higher CoVs for the ALSFRS-R. For example, the celecoxib study showed an ALSFRS-R CoV of 1.3 (3) and in a recent meta-analysis of creatine studies, the value was 1.26 (calculated from 186 patients overall) (24). The explanation for these inconsistencies is unclear, but could reflect inherent differences on a subjective measure such as the ALSFRS-R in a natural history study compared to an actual clinical drug trial (25).

This study had several limitations. First, the proportion of males to females was higher than expected at 3:1. The excess of males in this study was entirely coincidental, but does need to be considered since the proportion is not representative of the ALS population as a whole. Secondly, compared to most trials, our subjects enrolled after having been symptomatic for a relatively long time (30 months). This could have influenced the results of both our CoV analysis and the correlation to survival, although the effect is difficult to predict. Thirdly, we did not include a control population in this study. The underlying assumption was that all of the parameters being studied here, including EIM, should not change substantially over a severalmonth to one-year period of time in healthy individuals. Although we have not studied this question explicitly for EIM, an earlier reproducibility study did confirm stability 
of measurements in a group of 30 healthy individuals over an average of 113 days (range 7279 days) (12). Study of EIM over longer periods of time in normal subjects in a multicenter fashion is clearly warranted, as it would help establish the true inter-session repeatability. Fourthly, since the study continued for several years due to sluggish recruitment, at many sites the investigators performing the measurements changed, adding another source of potential variation, despite additional training. Finally, we did not evaluate bulbar and appendicular onset patients separately. Although the proportion of patients with primary bulbar onset in our study was only slightly below that of other recent studies, it remains possible that EIM may be less effective in this group of individuals and that a measure that incorporates bulbar dysfunction, such as the ALSFRS-R, would be more effective.

Nevertheless, nothing precludes performing EIM on such muscles, including the tongue; this remains an area for future study.

In summary, these results provide evidence that EIM can serve as a practical and sensitive method for the assessment of ALS progression in just six months. However, only by demonstrating its power to identify a treatment effect earlier and with fewer patients than standard measures will it be determined if EIM is actually also an effective biomarker of drug effect. The development of dedicated EIM devices (26) will help make it possible for ALS investigators to readily employ this technique to determine if that is the case.

\section{Acknowledgments}

This study was funded by the ALS Association. We thank Lindsay Garmirian, Connie Lin, Philip Mongiovi, Lucy Wang, Tuhin Srivastava, and Andrew Spieker for their assistance with this project.

Declaration of interest: S. B. Rutkove serves as a consultant and holds equity in Convergence Medical Devices, Inc. He has also receives consulting fees from Neuralstem, Inc., GlaxoSmithKline, Inc. and royalties from UpToDate. He also has two patent applications submitted in the field of electrical impedance. He receives grant funding from NIH, NASA, and the Spinal Muscular Atrophy Foundation. J. B. Caress receives grant funding from NIH.

M. S. Cartwright receives grant funding from NIH. T. Burns serves as Podcast Section Editor for Neurology ${ }^{\circledR}$; has served as a consultant for Bayhill Therapeutics; and has received research support from Knopp Neurosciences Inc. and the Myasthenia Gravis Foundation of America.

N. Maragakis serves on a scientific advisory board for Q Therapeutics, Inc.; received support from the Michael S. Ansari Gift Fund for basic science research; serves as a contributor to UpToDate; has served as a consultant for California Institute for Regenerative Medicine (CIRM); and receives/has received research support from Cytokinetics Inc., Sangamo BioSciences, Inc., TEDCO-Maryland Stem Cell Research Fund, NIH/NINDS, U.S. Department of Defense, and the ALS Association Packard Center for ALS Research at Johns Hopkins. M. Benatar has served as a consultant for Bayhill Therapeutics and Cytokinetics Inc.; receives publishing royalties for Neuromuscular Disease: Evidence and Analysis in Clinical Neurology (Humana Press, 2006), BluePrints in Neurology (Lippincott Williams \& Wilkins, 2002), and Field of Vision: A Manual and Atlas of Perimetry (Humana Press, 2010); receives/has received research support from CytRx Corporation, the Muscular Dystrophy Association, the ALS Association, the Food and Drug Administration, and the Centers for Disease Control and Prevention, the Woodruff Health Sciences Center (Emory University), and the NIH; and has participated in medico-legal cases. J. M. Shefner serves on a DSMB for the NIH; serves as Neuromuscular Section Editor for and receives publishing royalties from UpToDate; has served a consultant for Teva Pharmaceutical Industries Ltd., Gilead Sciences, Inc., GlaxoSmithKline, Trophos, and BrainGate; and receives research support from Isis Pharmaceuticals, Inc., Neuralstem, Inc., GlaxoSmithKline, Sanofi-Aventis, Teva Pharmaceutical Industries Ltd., Knopp Neurosciences Inc., Cytokinetics Inc., the NIH, and the ALS Association.

The authors alone are responsible for the content and writing of the paper.

\section{References}

1. Cudkowicz ME, Katz J, Moore DH, O’Neill G, Glass JD, Mitsumoto H, et al. Toward more efficient clinical trials for amyotrophic lateral sclerosis. Amyotroph Lateral Scler. 2010; 11:259265. [PubMed: 19961263] 
2. Cedarbaum JM, Stambler N, Malta E, Fuller C, Hilt D, Thurmond B, et al. The ALSFRS-R: a revised ALS functional rating scale that incorporates assessments of respiratory function. BDNF ALS Study Group (Phase III). J Neurol Sci. 1999; 169:13-21. [PubMed: 10540002]

3. Cudkowicz ME, Shefner JM, Schoenfeld DA, Zhang H, Andreasson KI, Rothstein JD, et al. Trial of celecoxib in amyotrophic lateral sclerosis. Ann Neurol. 2006; 60:22-31. [PubMed: 16802291]

4. Gordon PH, Moore DH, Miller RG, Florence JM, Verheijde JL, Doorish C, et al. Efficacy of minocycline in patients with amyotrophic lateral sclerosis: a phase III randomized trial. Lancet Neurol. 2007; 6:1045-1053. [PubMed: 17980667]

5. Aggarwal SP, Zinman L, Simpson E, McKinley J, Jackson KE, Pinto H, et al. Safety and efficacy of lithium in combination with riluzole for treatment of amyotrophic lateral sclerosis: a randomized, double-blind, placebo-controlled trial. Lancet Neurol. 2010; 9:481-488. [PubMed: 20363190]

6. Rutkove SB. Electrical Impedance Myography: Background, Current State, and Future Directions. Muscle Nerve. 2009; 40:936-946. [PubMed: 19768754]

7. Tarulli A, Esper G, Lee K, Aaron R, Shiffman C, Rutkove S. Electrical impedance myography in the bedside assessment of inflammatory myopathy. Neurology. 2005; 65:451-452. [PubMed: 16087913]

8. Rutkove SB, Shefner JM, Gregas M, Butler H, Caracciolo J, Lin C, et al. Characterizing spinal muscular atrophy with electrical impedance myography. Muscle Nerve. 2010; 42:915-921. [PubMed: 21104866]

9. Tarulli AW, Duggal N, Esper GJ, Garmirian LP, Fogerson PM, Lin CH, et al. Electrical impedance myography in the assessment of disuse atrophy. Arch Phys Med Rehabil. 2009; 90:1806-1810. [PubMed: 19801075]

10. Rutkove SB, Zhang H, Schoenfeld DA, Raynor EM, Shefner JM, Cudkowicz ME, et al. Electrical impedance myography to assess outcome in amyotrophic lateral sclerosis clinical trials. Clin Neurophysiol. 2007; 118:2413-2418. [PubMed: 17897874]

11. Wang LL, Spieker AJ, Li J, Rutkove SB. Electrical impedance myography for monitoring motor neuron loss in the SOD1-G93A amyotrophic lateral sclerosis rat. Clin Neurophysiol. 2011; 122:2505-2511. [PubMed: 21612980]

12. Rutkove SB, Lee KS, Shiffman CA, Aaron R. Test-Retest Reproducibility of $50 \mathrm{kHz}$ LinearElectrical Impedance Myography. Clin Neurophys. 2006; 117:1244-1248.

13. Shefner JM, Watson ML, Simionescu L, Caress JB, Burns TM, Maragakis NJ, et al. Multipoint incremental motor unit number estimation as an outcome measure in ALS. Neurology. 2011; 77:235-241. [PubMed: 21676915]

14. Li, J.; Fogerson, PM.; Rutkove, SB. Measuring the multifrequency electrical impedance of the mouse gastrocnemius muscle using a tetrapolar technique. International Conference on Electrical Impedance; April, 2010; Gainesville, FL. 2010. http://bioimpedance.bme.ufl.edu/icebi/2509_Rutkove.pdf.

15. Lungu C, Tarulli AW, Tarsy D, Mongiovi P, van der Horst VG, Rutkove SB. Quantifying muscle asymmetries in cervical dystonia with electrical impedance: a preliminary assessment. Clin Neurophysiol. 2011; 122:1027-1031. [PubMed: 20943436]

16. Ravits J, Paul P, Jorg C. Focality of upper and lower motor neuron degeneration at the clinical onset of ALS. Neurology. 2007; 68:1571-1575. [PubMed: 17485643]

17. Rutkove S, Partida R, Esper G, Aaron R, Shiffman C. Electrode position and size in electrical impedance myography. Clin Neurophys. 2005; 116:290-299.

18. Jaffrin MY. Body composition determination by bioimpedance: an update. Curr Opin Clin Nutr Metab Care. 2009; 12:482-486. [PubMed: 19494768]

19. Desport JC, Marin B, Funalot B, Preux PM, Couratier P. Phase angle is a prognostic factor for survival in amyotrophic lateral sclerosis. Amyotrophic lateral sclerosis: official publication of the World Federation of Neurology Research Group on Motor Neuron Diseases. 2008; 9:273-278. [PubMed: 18720089]

20. Cudkowicz ME, Shefner JM, Schoenfeld DA, Brown RH Jr, Johnson H, Qureshi M, et al. A randomized, placebo-controlled trial of topiramate in amyotrophic lateral sclerosis. Neurology. 2003; 61:456-464. [PubMed: 12939417] 
21. Brooks BR, Miller RG, Swash M, Munsat TL. El Escorial revisited: revised criteria for the diagnosis of amyotrophic lateral sclerosis. Amyotroph Lateral Scler Other Motor Neuron Disord. 2000; 1:293-299. [PubMed: 11464847]

22. Miller EG. Aymptotic test statistics for coefficient of variation. Comm in stats: theory and methods. 1991; 20:3351-3363.

23. Zhang Y, Schuff N, Woolley SC, Chiang GC, Boreta L, Laxamana J, et al. Progression of white matter degeneration in amyotrophic lateral sclerosis: a diffusion tensor imaging study. Amyotroph Lateral Scler. 2011; 12:421-429. [PubMed: 21745124]

24. Pastula DM, Moore DH, Bedlack RS. Creatine for amyotrophic lateral sclerosis/motor neuron disease. Cochrane Database Syst Rev. 2010 CD005225.

25. Chio A, Canosa A, Gallo S, Cammarosano S, Moglia C, Fuda G, et al. ALS clinical trials: do enrolled patients accurately represent the ALS population? Neurology. 2011; 77:1432-1437. [PubMed: 21956723]

26. Ogunnika OT, Rutkove SB, Ma H, Fogerson PM, Scharfstein M, Cooper RC, et al. A portable system for the assessment of neuromuscular diseases with electrical impedance myography. J Med Eng Technol. 2010; 35:377-385. [PubMed: 20670104] 


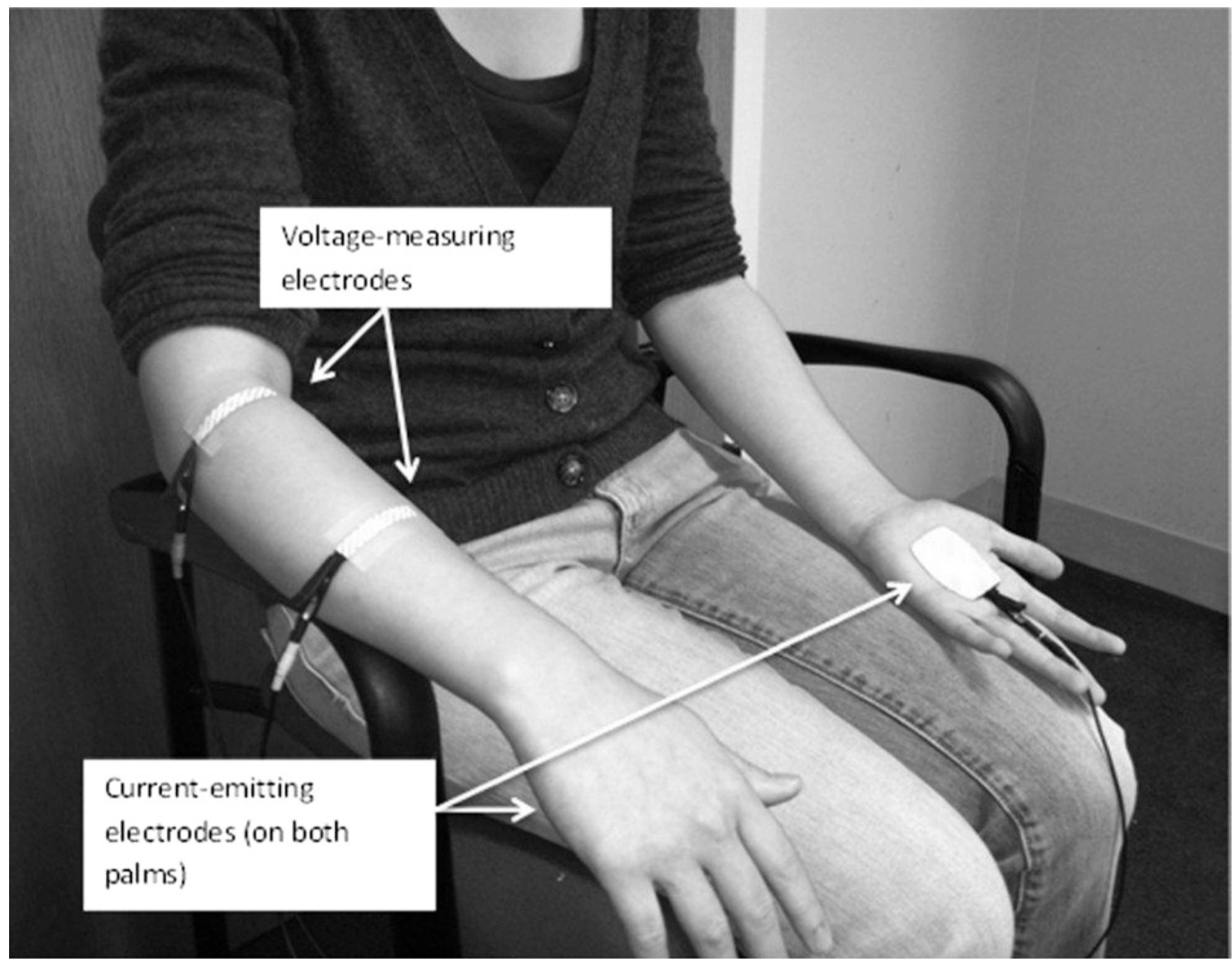

Figure 1.

Set-up for EIM on wrist extensors as performed in this study. The current-emitting electrodes are placed on each palm and the voltage-measuring electrodes are placed over the muscle of interest. The wires extend to the impedance-measuring device (not shown). In more recent work, this approach has been replaced by one in which the current-emitting electrodes are placed in close proximity to the voltage-measuring electrodes. 

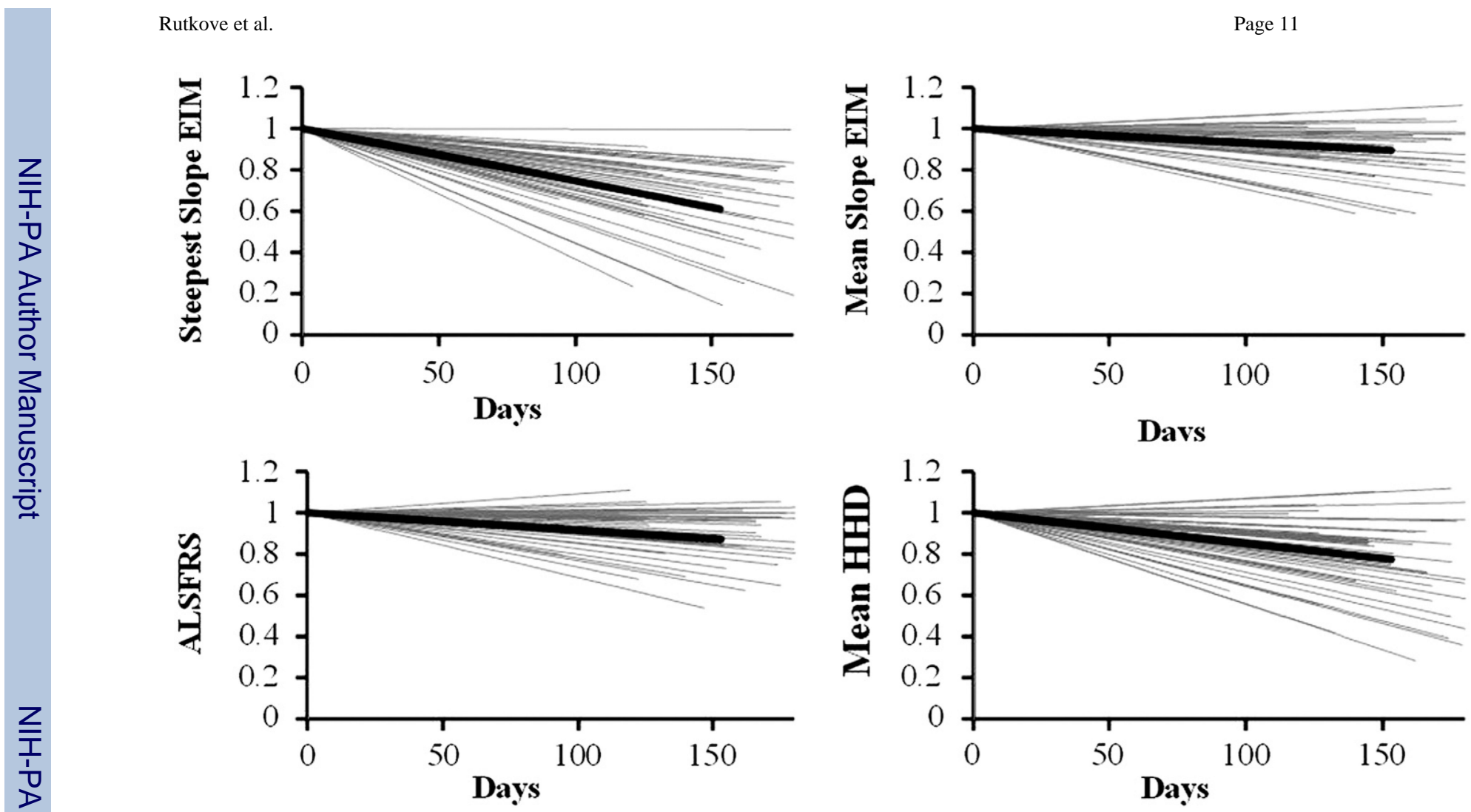

Figure 2.

Six-month relative trajectories for all 50 subjects for (a) EIM steepest slope, (b) EIM mean, (c) ALSFRS-R, and (d) HHD; mean value is in black, individual patient values in gray. 


\section{Table I}

Coefficients of variation in rate of decline at one year and at six months.

\begin{tabular}{lccccc}
\hline & \multicolumn{2}{c}{ One year } & & \multicolumn{2}{c}{ Six months } \\
\cline { 2 - 3 } \cline { 5 - 6 } $\begin{array}{l}\text { Outcome } \\
\text { measure }\end{array}$ & $\begin{array}{c}\text { Coefficient of } \\
\text { variation in } \\
\text { rate of decline }\end{array}$ & $\begin{array}{l}\mathbf{9 5 \%} \\
\text { confidence } \\
\text { interval }\end{array}$ & $\begin{array}{l}\text { Coefficient of } \\
\text { variation in } \\
\text { rate of decline }\end{array}$ & $\begin{array}{l}\mathbf{9 5 \%} \\
\text { confidence } \\
\text { interval }\end{array}$ \\
\hline EIM Phase-steepest slope & 0.62 & $0.47-0.77$ & & 0.55 & $0.41-0.69$ \\
EIM Phase-mean & 0.66 & $0.50-0.83$ & & 0.76 & $0.56-0.97$ \\
HHD & 0.82 & $0.59-1.0$ & & 0.92 & $0.63-1.2$ \\
ALSFRS-R & 0.74 & $0.55-0.93$ & 0.84 & $0.58-1.1$ \\
\hline
\end{tabular}




\section{Table II}

Number of patients needed per arm for a placebocontrolled trial*.

\begin{tabular}{lr}
\hline Outcome measure & Patients needed per study arm \\
\hline EIM steepest & 95 \\
EIM mean & 182 \\
HHD & 266 \\
ALSFRS-R & 220 \\
\hline \multirow{2}{*}{ * Assuming a six-month, placebo-controlled study, aiming to identify a 20\% treatment effect with $80 \%$ power, one-tailed, $p<0.05}$.
\end{tabular}




\section{Table III}

Correlation to survival.

\begin{tabular}{llcllc}
\hline & \multicolumn{2}{c}{ One year data } & & \multicolumn{2}{c}{ Six months data } \\
\cline { 2 - 3 } \cline { 5 - 6 } Outcome measure & $\begin{array}{l}\text { Cox hazards ratio } \\
\mathbf{( 9 5 \%} \mathbf{~ C L})\end{array}$ & $p$-value & & $\begin{array}{l}\text { Cox hazards ratio } \\
\mathbf{( 9 5 \%} \text { CL) }\end{array}$ & $p$-value \\
\hline EIM Phase-steepest & $1.68[1.21-2.32]$ & 0.0018 & & $1.40[1.02-1.90]$ & 0.035 \\
EIM Phase-mean & $1.02[0.73-1.44]$ & 0.903 & & $0.89[0.59-1.36]$ & 0.59 \\
HHD & $2.47[1.64-3.72]$ & $<0.001$ & & $1.93[1.30-2.87]$ & 0.0012 \\
ALSFRS-R & $5.81[2.68-12.6]$ & $<0.001$ & & $2.49[1.47-4.22]$ & $<0.001$ \\
\hline
\end{tabular}

Values given as mean +/- standard deviation; CL: confidence limit. 